\title{
Analytical RTT Estimation of a 3-D Elevator System by Exact Stop Positions - Extension to Multi-floor and Non-uniform Population Applications
}

\author{
Albert So \\ Faculty of Arts, Science and Technology, The University of Northampton, U.K., \\ alberttpso@gmail.com
}

\begin{abstract}
3-dimensional (3-D) elevator systems will be the industrial trend. Traditionally, designers first perform a calculation on up-peak traffic analysis to get an overall concept and then proceed to carry out computer simulation to obtain details under different traffic patterns. This tradition is still maintained throughout the world. Previously, a "Scanner" approach together with a sophisticated origin/destination matrix, were adopted to calculate the round trip time $(R T T)$ of the 3-D system. In a more recent article, by using Order Statistics, a series of repeatable stops in a 3-D system was broken down into several series of non-repeatable stops to estimate the $R T T$. However, a uniform population distribution of all potential stops and a single floor service were assumed in that latest article. The development in this article has been built upon the two previous articles to extend the up-peak $R T T$ calculation to a more generic approach, including non-uniform population distribution of stops, and multi-floor applications. The latter also deals with the existence of multiple vertical risers. This new development has been validated by Monte Carlo simulation, which may be the most general approach to calculate the up-peak RTT of a truly 3-D elevator system, and to be followed by computer simulation to implement the traditionally industrial practice in the three dimensional world.
\end{abstract}

Keywords: Traffic analysis, 3-dimensional elevator system, round trip time, non-uniform population, multi-floor service.

\section{$1 \quad$ INTRODUCTION}

Following the successful development of the world's first prototype of a 2-dimensional (2-D) elevator in Germany [1], 2-D or even 3-D elevator systems will soon become popular around the world [2] because buildings tend to become taller and wider. The author, with others, first developed methods to calculate the round trip time (RTT) of a 2-D elevator system [3-5].

Then, it comes to a 3-D elevator system. Fig. 1 and Fig. 2 show the configuration where a 3-D elevator system is considered feasible [6]. During an up-peak period, passengers arrive at the main terminal of a building where there are a number of cars available, say five, E1 to E5, as shown in the Fig. 2. A destination group control system (DGS) is to be adopted. The system gathers passengers whose destination is the same floor together and signals them to board a designated car. This is because it is technically more efficient for an elevator car to move horizontally rather than vertically. 


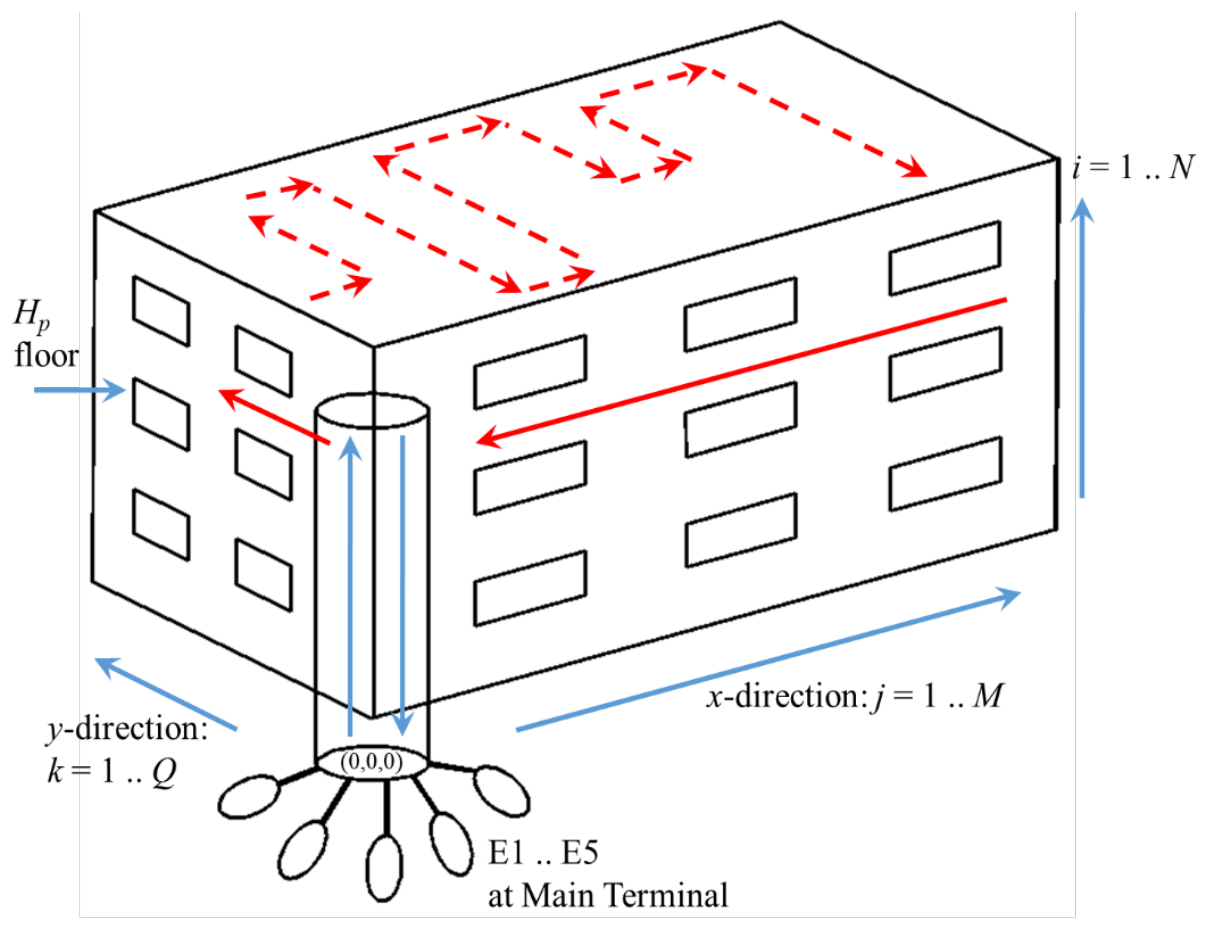

Figure 1 A typical 3-dimensional elevator system

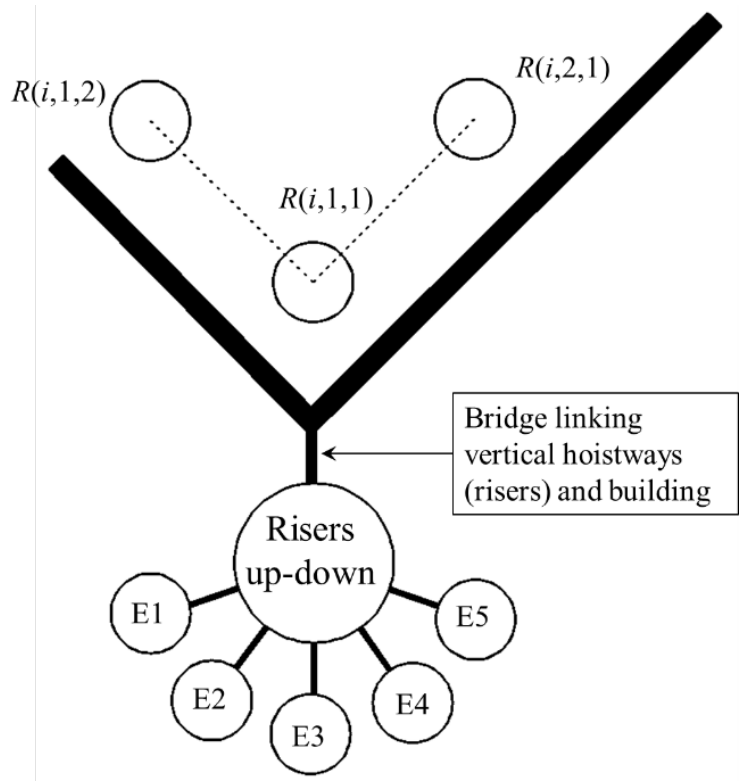

Figure 2 Zoomed view of the common hoistways

After the car is fully loaded, it leaves the main terminal and enters the vertical riser where there could be several vertical hoistways. The minimum number is three, one for up, one for down and one spare for either direction of movement in the case of emergency. The advantage of one 
hoistway for one direction of movement is to avoid jamming along any hoistway. Once the car gets to the destination floor, the $i$ th floor, it travels to the first potential stop, $R(i, 1,1)$ via a bridge linking every floor to the vertical hoistways. If some passengers want to exit the car at this stop, the doors will be opened. Then, the car travels to the second stop.

The "Scanner" model is illustrated by Table 1. Without loss of generality, every floor consists of $M \times Q$ ( $M$ along the $x$-axis, i.e. the columns, and $Q$ along the $y$-axis, i.e. the rows) potential stops. A potential stop is a real physical stopping position of the elevator with landing doors. The scanning path is along a column first and then incremented by one row.

Therefore, the first potential stop is indicated by $R(i, 1,1)$, second by $R(i, 1,2)$, until the $Q$ th stop by $R(i, 1, Q)$. Here, $Q$ is called the scanned sequence number while $(i, 1, Q)$ is the physical co-ordinates of that stop. The $(Q+1)$ th stop is indicated by $R(i, 2, Q)$ and the $(Q+2)$ th stop by $R(i, 2, Q-1)$ and so on until the last potential stop indicated by $R(i, M, Q)$. Without loss of generality, it is assumed that $M$ is always an odd integer, meaning that the last stop is always at the other end of the diagonal with $R(\mathrm{i}, 1,1)$ at one end. A potential stop on a floor is equivalent to a floor of a 1-D system in a building in terms of the scanned sequence number. Each potential stop, being a real and physical stop, represents a group of occupants whose offices are in the vicinity of, i.e. within a reasonable walking distance from, the potential stop. In the previous articles, all stops were assumed to be uniformly populated. For the rest of this article, " $R$ " of each stop will be neglected and only the coordinates are indicated. 
Table 1 Stops arrangement and the sequence of serving the stops (scanner method)

\begin{tabular}{|c|c|c|c|c|c|c|c|c|c|c|}
\hline$Q$ & $Q+1$ & $3 Q$ & $3 Q+1$ & $5 Q$ & $?$ & $\cdot$ & $\%$ & \begin{tabular}{|cc}
$\cdot$ & \\
\end{tabular} & $(M-2) \cdot Q+1$ & $M . Q$ \\
\hline$Q-1$ & $Q+2$ & $3 Q-1$ & $3 Q+2$ & $\sqrt[5 Q-1]{5}$ & . & . & . & . & $(M-2) \cdot Q+2$ & $M \cdot Q-1$ \\
\hline . & . & . & . & . & . & . & . & . & . & . \\
\hline . & . & . & . & & $\cdot$ & . & $\cdot$ & . & . & . \\
\hline 2 & $2 Q-1$ & $2 Q+2$ & $4 Q-1$ & $4 Q+2$ & 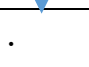 & . & . & . & $(M-1) \cdot Q-1$ & $(M-1) \cdot Q+2$ \\
\hline 1 & $2 Q$ & $2 Q+1$ & $4 Q$ & $4 Q+1$ & & $\phi$ & . & $\rightarrow$ & $(M-1) \cdot Q$ & $(M-1) \cdot Q+1$ \\
\hline
\end{tabular}

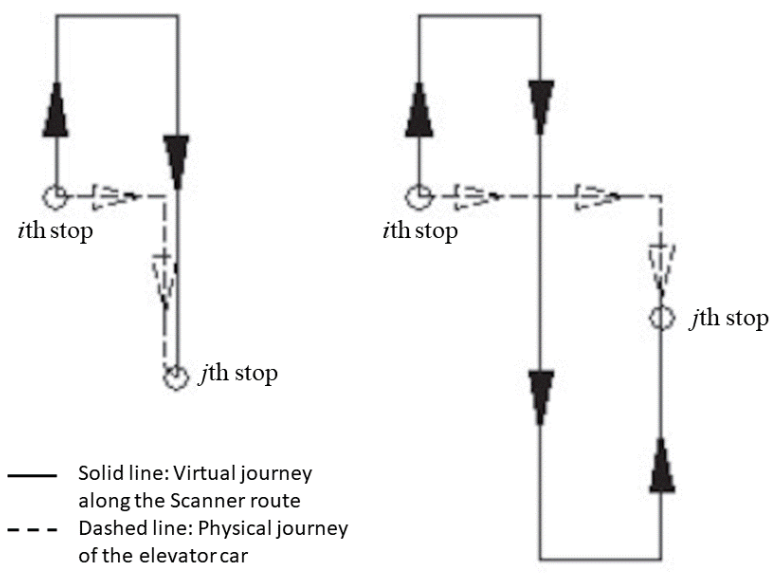

Figure 3 Physical path of motion of the elevator car

Although the scanning path is based on Table 1 meaning that the controller always handles a stop with a scanned sequence number smaller than that of the next stop. But once a series of real stops has been confirmed, the actual path of motion of the elevator car is according to that shown in Fig. 3. The solid line in Fig. 3 shows the scanning route with incremental scanned sequence number while the dotted line shows the physical route of movement. It can be seen that there could be up to one $90^{\circ}$ turn from the $i$ th stop to the next stop, i.e. the $j$ th stop, $i$ and $j$ being the scanned sequence numbers. When two consecutive stops happen to be on the same row or same column, no $90^{\circ}$ turn is necessary. The simulation considers the kinematics of the car to travel from one stop stationary to the next stop stationary. But in the calculation, for simplicity, an ideal motion involving constant acceleration, rated speed and deceleration is assumed.

In the previous article [6], a probability matrix from any origin stop, i.e. the " $s$ " stop, to the destination stop, i.e. the " $e$ " stop, on the $i$ th floor, was generated as shown in Table 2. 
Table 2 The journey probability matrix at a floor (the $i$ th floor in this case)

\begin{tabular}{|c|c|c|c|c|c|c|c|}
\hline & 1 & 2 & 3 & $\ldots$ & MQ-2 & MQ-1 & $M Q$ \\
\hline$\overline{\overline{1}}$ & 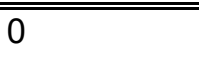 & $\overline{P P\left(i, J_{12}\right)}$ & $\overline{P P\left(i, J_{13}\right)}$ & $\ldots$ & 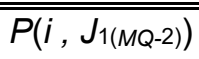 & $\overline{P P\left(i, J_{1(M Q-1)}\right)}$ & 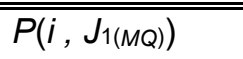 \\
\hline 2 & $P\left(i, J_{21}\right)$ & 0 & $P\left(i, J_{23}\right)$ & $\ldots$ & $P\left(i, J_{2(M Q-2)}\right)$ & $P\left(i, J_{2(M Q-1)}\right)$ & $P\left(i, J_{2(M Q)}\right)$ \\
\hline 3 & $P\left(i, J_{30}\right)$ & 0 & 0 & $\ldots$ & $P\left(i, J_{3(M Q-2)}\right)$ & $P\left(i, J_{3(M Q-1)}\right)$ & $P\left(i, J_{3(M Q)}\right)$ \\
\hline$\cdots$ & $\cdots$ & $\cdots$ & $\ldots$ & 0 & $\ldots$ & $\cdots$ & $\ldots$ \\
\hline$M Q-2$ & $P\left(i, J_{(M Q-2) 1}\right)$ & 0 & 0 & $\ldots$ & 0 & $P\left(i, J_{(M Q-2)}(M Q-1)\right)$ & $P\left(i, J_{(M Q-2)(M Q)}\right)$ \\
\hline$M Q-1$ & $P\left(i, J_{(M Q-1) 1}\right)$ & 0 & 0 & $\ldots$ & 0 & 0 & $P\left(i, J_{(M Q-1)(M Q)}\right)$ \\
\hline$M Q$ & $P\left(i, J_{(M Q) 1}\right)$ & 0 & 0 & $\ldots$ & 0 & 0 & 0 \\
\hline
\end{tabular}

The reason why most entries below the "( $(1,1)-(M Q, M Q)$ " diagonal are equal to zero is that the car only moves forward along the scanning route with incremental scanned sequence numbers and the car cannot move to its current position. Only after the last passenger has been served and the car gets vacant that the car returns to $R(i, 1,1)$ to get into the vertical hoistways to express down the main terminal. That explains why entries along the first column are nonzero. The average $R T T$ of that particular floor is then estimated by multiplying the probability of a journey from the $s$ stop to the $e$ stop by the journey time needed without any stop in between and then summing up all such products of all stop pairs on the $i$ th floor, as shown in Eq. 1.

$$
\begin{gathered}
\left.P\left(i, J_{s e}\right)\right|_{e>s}=\left(1-\sum_{c=s+1}^{e-1}\left(\frac{U(i)_{c}}{U(i, 0,0)}\right)\right)^{P}-\left(1-\sum_{c=s}^{e-1}\left(\frac{U(i)_{c}}{U(i, 0,0)}\right)\right)^{P} \\
-\left(1-\sum_{c=s+1}^{e}\left(\frac{U(i)_{c}}{U(i, 0,0)}\right)\right)^{P}+\left(1-\sum_{c=s}^{e}\left(\frac{U(i)_{c}}{U(i, 0,0)}\right)\right)^{P} \\
P\left(i, J_{0 e}\right)=\left(1-\sum_{c=1}^{e-1}\left(\frac{U(i)_{c}}{U(i, 0,0)}\right)\right)^{P}-\left(1-\sum_{c=1}^{e}\left(\frac{U(i)_{c}}{U(i, 0,0)}\right)\right)^{P} \\
P\left(i, J_{s 0}\right)=\left(\sum_{c=1}^{s}\left(\frac{U(i)_{c}}{U(i, 0,0)}\right)\right)^{P}-\left(\sum_{c=1}^{s-1}\left(\frac{U(i)_{c}}{U(i, 0,0)}\right)\right)^{P}
\end{gathered}
$$

where $U(i)_{c}$ is the population of room " $c$ " (either " $s$ " or " $e$ ") on the $i$ th floor, $U(i, 0,0)$ is the total floor population of the $i$ th floor, and $P$ is the number of passengers entering the car at the main terminal.

This approach can really provide a solution to the computation of the RTT of such a 3-D system. However, there are two shortcomings. First, the matrix is well too complicated with more than $(1 / 2) M^{2} Q^{2}$ meaningful elements, in particular, when $M$ and $Q$ are relatively large. Second, Eq. 1 lumps all expressions together into one expression while the designers cannot fully understand what happens inside. Thus, variation of parameters to modify a design during the design stage becomes difficult. 
After this model was developed, further work was carried out on using graphical methods in order to resolve potential collision between consecutive elevators travelling along the same hoistway [7]. Some further work on finding the optimal route of a combination of car-calls on the destination floor by using deep reinforcement learning involving artificial neural networks (ANNs) was also conducted [8].

\section{RTT COMPUTATION BY EXACT STOPPING LOCATION IDENTIFICATION}

To avoid handling such huge and complicated probability matrix and to help designers follow a step-by-step process by having a clear understanding what happens in the whole process of estimation and in the mechanism to perform sensitivity analysis or vary design parameters, like that in the traditional process for a 1-D system, a new method based on a branch of mathematics, Order Statistics, was developed [9]. In that article, the assumption of a uniformly populated building was also made to simplify certain derivation of formulae.

In the conventional up-peak (uniform population distribution) traffic calculation of a 1-D system, as shown in Eq. 2, $H$ (highest reversal floor), $L$ (lowest arrival floor) and $S$ (expected number of stops in one round trip) are usually non-integral. That does not matter in estimating the $R T T$ of a 1-D system. But in a 3-D system, the RTT is very sensitive to the exact stopping positions because the scanning route is not the same as the moving route of the elevator, as shown in Fig. 3.

$$
\begin{aligned}
& R T T=2 H t_{v}+(S+1)\left(T-t_{v}\right)+2 P t_{p} \\
& S=N\left[1-\left(\frac{N-1}{N}\right)^{P}\right] ; H=N-\sum_{i=1}^{N-1}\left(\frac{i}{N}\right)^{P} ; \\
& L=1+\sum_{i=1}^{N-1}\left(\frac{i}{N}\right)^{P}
\end{aligned}
$$

where

$H=$ highest stop of reversal on average;

$L=$ lowest floor of the first stop on average;

$S=$ expected number of stops;

$t_{v}=$ time of one floor jump under rated speed;

$N=$ number of floors above main terminal;

$T=$ performance time; $P=$ number of passengers in the elevator car;

$t_{p}=$ passenger transfer time.

The reason why non-integral values exist is that they are statistical figures. For $P$ number of passengers inside a car, there can be one stop, two stops, up to $P$ number of stops, even within one round trip. These are termed "repeatable stops". To accurately calculate the time taken for one round trip in a 3-D system, one needs to identify the exact stopping positions and the exact number of stops within each trip because time taken for the car to move between stops is very sensitive to the exact location of stops. This is based on finding out the average travel time 
between non-repeatable stopping positions and the final $R T T$ is the weighted average of all of them. When stops are non-repeatable, and hence integral numerically, stopping positions can be exactly defined and traced. The most recent article [9] provides a solution to break down a series of repeatable stops into several series of non-repeatable stops while the $R T T$ of each series of non-repeatable stops can be calculated precisely and accurately. Then, the total RTT can be calculated by the sum of all such "non-repeatable" RTTs weighted by their respective probability density functions. It was shown that the $R T T$ obtained was identical to each other by the two approaches, i.e. "Monte Carlo simulation" with repeatable stops and "Calculation based on Order Statistics" with non-repeatable stops.

In [9], two assumptions were made, first all potential stops being uniformly populated and second, only one floor being served in every round trip during the up-peak period. In this article, such two assumptions are removed and the method "Calculation based on Order Statistics" can be extended to a more generic 3-D up-peak traffic analysis.

\section{RTT ESTIMATION OF A 3-D ELEVATOR SYSTEM BY WEIGHTED SUM OF RTT'S WITHOUT REPETITION (NON-UNIFORM POPULATION AND MULTIPLE FLOORS)}

The method adopted in [9] is briefly explained here for completeness and quick reference. Conceptually, $P$ number of passenger stops with repetition may actually be viewed as consisting of a combination of 1 number of stop, 2 number of stops without repetition, 3 number of stops without repetition, until $P$ number of stops without repetition. Here, "with repetition" means that the exact physical number of stops of the $P$ passenger may be any number equal to or smaller than $P$. But "without repetition" means that the number of physical stops is exactly equal to $P$. One important assumption here is that all stops must be equally likely, i.e. uniform population density of stops.

For example, suppose $N=6$ and $P=3$. In every up-peak journey, there could be up to $N^{P}$ possible combinations of stops for the $P$ passengers, i.e. $6^{3}=216$. If these combinations are arranged in an ascending order, there could be three types where the first digit represents the destination of the first passenger, second digit the second passenger, and so on, and they are shown below.

i) one stopping position only, say " $2,2,2$ ", “3,3,3" or " $6,6,6$ " etc. and there are 6 possibilities and they are all considered "one stop without repetition";

ii) two stopping positions, say " $1,1,2$ ", "2,5,5", "4,4,6" etc.; " $1,1,2$ " may come from " $2,1,1$ ", " $1,2,1$ " or " $1,1,2$ ", and they are all considered "two stops without repetition"; both " $1,1,2$ " and " $2,1,1$ " refer to " 1,2 " without repetition;

iii) three stopping positions, say " $1,2,3$ ", " $2,4,5$ " etc.; " $1,2,3$ " may come from " $3,2,1$ ", “ $3,1,2$ ", " $2,3,1$ ”, " $2,1,3$ ", " $1,3,2$ " or " $1,2,3$ ", and they are all considered "three stops without repetition", i.e. "1,2,3".

All possibilities of types (i), (ii) and (iii) should sum up to 216 . The next step is to estimate the probability density function of each type of stops without repetition. Let's denote $S^{N}{ }_{n}(k)$ as the series of stopping positions in an ascending order, where $N=$ total number of possible floors or stops allowed, $n=$ types of stops, $n=1, \ldots, P$, and $k=$ the passenger stopping sequence number 
within the series, $k=1, \ldots, n$. Since the 3 -D system has already been linearized into a 1-D system, $N$ could refer to the total number of possible stops or possible floors. For example, in " $2,5,5$ " of type $2, S_{2}^{6}{ }_{2}(1)=2$ nd floor and $S^{6}{ }_{2}(2)=5$ th floor, and similar for others because " $2,5,5$ " is one particular combination of " 2,5 ". And $\operatorname{Pr}\left(S^{N}{ }_{n}\right)$, shown in Eq. 3, is the probability density function of the $n$th type of stops which is equal to the number of all possible combinations of $n$ stops without repetition divided by $N^{P}$.

For each possibility, $\sum_{i=1}^{n} a(i)=P$ and $\forall i: \quad 1 \leq a(i) \leq P-n+1$

$\operatorname{Pr}\left(S_{n}^{N}\right)=\frac{\left({ }_{N} C_{n} \cdot \sum_{\begin{array}{c}\forall a(i) \\ \text { combinations }\end{array}} \frac{P !}{\prod_{i=1}^{n} a(i) !}\right)}{N^{P}}$

In a 3-D system, the calculation of $R T T$ in Eq. 2 is not directly applicable because the $R T T$ is very sensitive to actual stopping positions due to the nonequivalence between the scanning route and the moving route. Moreover, $H, L$ and $S$ are all non-integral in Eq. 2, making the estimation of journey time between stops impossible in a 3-D system.

The $P$ stops with repetition can be broken down into series of 1 (the $S^{N}{ }_{1}$ scenario), 2 (the $S^{N_{2}}$ scenario), $3, \ldots$, up to $P$ (the $S^{N}{ }_{P}$ scenario) stop(s) without repetition. And the exact $R T T$ of each type of stops without repetition, for $1,2,3, \ldots, P$ stops, can be analytically estimated as follows. And the final $R T T, R T T($ all $)$, of the $P$ stops with repetition can be found by the weighted sum of all these types of stops, as shown in Eq. 4.

$R T T($ all $)=\sum_{n=1}^{P} R T T\left(S_{n}^{N}\right) \cdot \operatorname{Pr}\left(S_{n}^{N}\right)$

To compute $\operatorname{RTT}\left(S^{N}\right)$, as the round trip is extended to multiple floors in this article, the following components are to be considered. The $n$ stops consist of the 1st, 2nd, .., $k$ th, $(k+1)$ th, $\ldots,(n-1), n$th stop. $i, j \in\{1, \ldots, M . Q\}$ are scanned sequence number of possible stops on the horizontal plane of one or more floors. If two floors are included in one round trip, $N$ becomes $2 M Q$. Physically, an elevator car must depart from the $k$ th stop within the $n$ stops ( physically the $i$ th stop at $(x(i), y(i))$ to the $(k+1)$ th stop within the $n$ stops ( physically the $j$ th stop at $(x(j), y(j))$.

The actual path of motion from the $i$ th stop to the $j$ th stop is shown in Fig. 3, i.e. from $(x(i)$, $y(i))$ to $(x(j), y(i))$, and finally to $(x(j), y(j))$. Total time of such a journey between two consecutive passenger stops in our simulation is the sum of the following components with simplification, altogether called tij $(x(i), y(i), x(j), y(j))$. But in a practical sense, the comfortable kinematics of the horizontal motion of the car has to be taken care of where jerk, acceleration and deceleration have to be considered.

i) $t_{c}:$ door closing time; 
ii) $0.5 t_{h}(1)$ : time for horizontal acceleration for a distance usually equal to half of the separation, $d_{f}$, between two consecutive physical stops on the floor;

iii) $\left(|x(j)-x(\mathrm{i})|-d_{f}\right) / v_{h}$ or $\left(|y(j)-y(i)|-d_{f}\right) / v_{h}$ : time for horizontal rated speed operation where $v_{h}$ is the horizontal rated speed of the elevator car; in our simulation, the elevator car always moves along a row first and then along a column;

iv) $0.5 t_{h}(1):$ time for horizontal deceleration to the intermediate stop to change its direction of motion;

v) $t_{T H H}$ : time for the elevator car to turn $90^{\circ}$ between two horizontally perpendicular hoistways;

vi) $0.5 t_{h}(1):$ time for horizontal acceleration for a distance usually equal to half of the separation, $d_{f}$, between two consecutive physical stops on the floor;

vii) $\left(|x(j)-x(\mathrm{i})|-d_{f}\right) / v$ or $\left(|y(j)-y(i)|-d_{f}\right) / v$ : time for horizontal rated speed operation where $v$ is the horizontal rated speed of the elevator car;

viii) $0.5 t_{h}(1)$ : time for horizontal deceleration to the final stop;

ix) $t_{o}$ : door opening time.

If both origin and destination stops are along the same row or column, half of the steps above can be cancelled. Without loss of generality, a 2-consecutive-floor round trip is considered where there are $n_{L}$ real stops on the lower floor and $n_{H}$ real stops on the higher floor, $n_{L}+n_{H}=$ $n$ made by the car within a particular round trip. But this method is not limited to a round trip to two floors only and these multiple floors may not necessarily be contiguous. The estimation of total RTT involves five types of journeys.

i) The first type is from the main terminal at the ground floor to the first stop on the lower floor, called a "riser-up" journey.

ii) The second type is between each pair within the $n_{L}$ stops on the lower floor, i.e. 1st to 2 nd, 2 nd to $3 \mathrm{rd}, \ldots,(k-1)$ th to $k$ th, $k$ th to $(k+1)$ th, ..., $\left(n_{L}-1\right)$ th to $n_{L}$ th, called "intrafloor" journeys on the lower floor.

iii) The third type is from the $n_{L}$ th stop on the lower floor to the first stop on the upper floor, called an "interfloor" journey. That involves a travel from the $n_{\mathrm{L}}$ th stop to a riser, from the riser at the lower floor to the riser at the higher floor, and finally from the riser to the first stop on the upper floor. The original design involves one column of vertical hoistways only, close to the main terminal. And in this article, another option involving two columns at two opposite corners along the diagonal of the building is also considered.

iv) The fourth type is between each pair within the $n_{H}$ stops on the higher floor, i.e. from the first stop on the upper floor, until the last stop, i.e. the $n_{H}$ th stop on the higher floor, also a type of "intrafloor" journeys.

v) The fifth type is from the $n_{H}$ th stop on the higher floor to the main terminal at the ground floor, called a "riser-down" journey.

Computationally, (i) is equivalent to (v), and (ii) is equivalent to (iv). Although only two floors are considered, there is no reason why the method cannot be extended to three or more floors 
by breaking up $n$ into more fractions. But practically, it takes long for an elevator car to move vertically between floors and therefore, the efficiency is rather low if more than two floors are involved in one round trip. The details are shown below. Derivation of the following equations may refer to [9].

\section{a) Riser-up Journey $\left(t_{\text {ruj }}\right)$ to the lower floor}

The first stop can be any one of the first $(N=M \cdot Q)-n_{L}+1$ possible stops on the floor along the "Scanner route". The travel time consists of the following elements:

i) from main terminal to the lower floor level along the riser, which is neglected in this study as it is always a constant for all round trips involving this lower floor as the first destination;

ii) $\quad r s t$ : from the riser at the lower floor level to the stop $(x(1), y(1))$ closest to the riser including acceleration and deceleration;

iii) $t_{T H H}$ : stopping time at stop $(x(1), y(1))$ to change direction of travel, preparing for further journey to the first passenger stop;

iv) tij : journey time from $(x(1), y(1))$ to the first passenger stop; this part is neglected if $(x(1), \mathrm{y}(1))$ is the first passenger stop by itself.

The probability of each stop is simply $1 /\left(N-n_{L}+1\right)$. Hence the weighted sum of this journey, $t_{r u j}$, is given by Eq. 5 .

$t_{r u j}=r s t+t_{T H H}+\sum_{i=1}^{N-n_{L}+1} \frac{1}{N-n_{L}+1} t i j(x(1), y(1), x(i), y(i))$

\section{b) Intrafloor journey $\left(t_{i j j}\right)$ on the lower floor}

If there is only one passenger stop for the floor, i.e. $n_{L}=1$, this part is neglected. So, the computation of $t_{i f j}$ is only meaningful if $n_{L}=2$ or more. It starts from the first passenger stop to the second passenger stop and then from the second to the third and finally to the $n_{L}$ th stop. Let's assume the $a$ th passenger stop is the $i$ th stop and the $(a+1)$ th stop is the $j$ th stop, $i$ and $j$ being the scanned sequence number on the lower floor, and $a=1, \ldots, n_{L}-1$. The travel time from the $i$ th stop to the $j$ th stop, is simply given by $\operatorname{tij}(x(i), y(i), x(j), y(j))$. But the probability is a bit more complicated.

Before the $i$ th stop, there are ( $a$-1) stops distributed within $(i-1)$ possible stops and after the $j$ th stop, there are $\left(n_{\mathrm{L}}-(a+1)\right)$ stops distributed within the remaining $(N-j)$ stops. Total possible combinations $={ }_{(i-1)} C_{(a-1)} \cdot{ }_{(N-j)} C_{(n L-(a+1))}$. The $a$ th stop can correspond to either of $i=a, i=a+1$, $i=a+2$ until $i=a+N-n_{L}$ while the $(a+1)$ th stop can correspond to either one of $j=a+1, j=a+2$ until $j=a+N-n_{L}+1$. Hence, the average intrafloor journey time, $t_{i f j}(a, a+1)$ from the $a$ th stop to the $(a+1)$ th stop is given by Eq. 6 . 
$t_{i j j}(a, a+1)=\sum_{i=a}^{a+N-n_{L}} \sum_{j=i+1}^{a+N-n_{L}+1} t i j(x(i), y(i), x(j), y(j)) \frac{C_{a-1}^{i-1} \cdot C_{n_{L}-(a+1)}^{N-j}}{C}$

where $C=\sum_{i=a}^{a+N-n_{L}} \sum_{j=i+1}^{a+N-n_{L}+1} C_{a-1}^{i-1} \cdot C_{n_{L}-(a+1)}^{N-j}$

The intrafloor journey time for other pairs can be handled similarly.

c) Interfloor journey from the lower floor to the higher floor $\left(t_{i t f j}\right)$

i) tij: journey from the last passenger stop, i.e. the $n_{L}$ th stop, to either the first possible stop $(x(1), y(1))$ or $(x(M Q), y(M Q))$ depending on the location of the vertical riser; this part is neglected if $(x(M Q), y(M Q))$ is the last passenger stop on the lower floor while the riser close to $(x(M Q), y(M Q))$ is chosen;

ii) $t_{T H H}$ : stopping time at either $(x(1), y(1))$ or $(x(M Q), y(M Q))$ to change direction of travel;

iii) $\quad r s t$ : journey from either $(x(1), y(1))$ or $(x(M Q), y(M Q))$ to the riser on the lower floor, both being the same;

iv) tlfhf: time to travel from the lower floor level to the higher floor level along the riser, including acceleration, rated speed movement and deceleration; this is computed by $t_{T H V}$ (stopping time at the riser to change from a horizontal movement to a vertical movement), $t_{v}(1)$ (acceleration and deceleration time along a vertical hoistway) and $v_{v}$ (vertical rated speed);

v) $r s t$ : journey from the riser at the higher floor level to either $(x(1), y(1))$ or $(x(M Q)$, $y(M Q))$ on the higher floor;

vi) $t_{T H H}$ : stopping time at either $(x(1), y(1))$ or $(x(M Q), y(M Q))$ to change direction of travel;

vii) tij: journey from the first possible stop on the higher floor, either $(x(1), y(1))$ or $(x(M Q)$, $y(M Q))$ to the first passenger stop on the higher floor, depending on the location of the rise; this part is neglected if either $(x(1), y(1))$ or $(x(M Q), y(M Q))$ is the first passenger stop by itself.

The weighted sum of this journey, $t_{i t f j}$, is given by Eq. 7. There are two different expressions, depending on the exact location of the riser used. If the riser close to $(x(1), y(1))$ is the only riser, there is no need to change the scanned sequence numbering process. That shown in Table 1 is good enough. However, if the second option of installing another vertical riser close to $(x(M Q), y(M Q))$ on the lower floor is adopted, the scanning and numbering process on the higher floor has to be reversed, i.e. $i$ is replaced by $(N=M Q)-i+1$. 
If riser is close to $(x(N), y(N))$ on the lower floor, $N=M Q$

$$
\begin{aligned}
t_{i t f j}= & 2 r s t+2 t_{T H H}+t l f h f+\sum_{i=n_{L}}^{N} \frac{1}{N-n_{L}+1} t i j(x(i), y(i), x(N), y(N)) \\
& +\sum_{i=1}^{N-n_{H}+1} \frac{1}{N-n_{H}+1} t i j(x(1), y(1), x(i), y(i))
\end{aligned}
$$

If riser is close to $(x(1), y(1))$ on the lower floor, $N=M Q$

$$
\begin{aligned}
t_{i f f j}= & 2 r s t+2 t_{T H H}+t l f h f+\sum_{i=n_{L}}^{N} \frac{1}{N-n_{L}+1} t i j(x(i), y(i), x(1), y(1)) \\
& +\sum_{i=1}^{N-n_{H}+1} \frac{1}{N-n_{H}+1} t i j(x(1), y(1), x(i), y(i))
\end{aligned}
$$

\section{d) Intrafloor journey $\left(t_{i f j}\right)$ on the higher floor}

The whole procedure is similar to that in sub-section (b) provided that the scanning and numbering process is proper. Again, the time taken to travel from the $a$ th passenger stop to the $(a+1)$ th passenger stop is shown in Eq. 8.

$t_{i f j}(a, a+1)=\sum_{i=a}^{a+N-n_{H}} \sum_{j=i+1}^{a+N-n_{H}+1} t i j(x(i), y(i), x(j), y(j)) \frac{C_{a-1}^{i-1} \cdot C_{n_{H}-(a+1)}^{N-j}}{C}$

where $C=\sum_{i=a}^{a+N-n_{H}} \sum_{j=i+1}^{a+N-n_{H}+1} C_{a-1}^{i-1} \cdot C_{n_{H}-(a+1)}^{N-j}$

The intrafloor journey time for other pairs on the higher floor can be handled similarly.

\section{e) Riser-down journey $\left(t_{r d j}\right)$ from the higher floor}

This is similar to (a). The last passenger stop can be any one of the last $(N=M \cdot Q)-n_{H}+1$ possible stops. The travel time consists of the following elements:

i) tij : journey from the last passenger stop back to $(x(1), y(1))$;

ii) $t_{T H H}$ : stopping time at stop $(x(1), y(1))$ to change direction of travel, preparing for further journey to the riser;

iii) $\quad r s t$ : from stop $(x(1), y(1))$ to the riser including acceleration and deceleration;

iv) from the higher floor back to the main terminal along the riser, which is neglected in this study as it is always a constant for the same destination floor.

The probability of each stop is simply $1 /\left(N-n_{H}+1\right)$. Hence the weighted sum of this journey, $t_{r d j}$, is given by Eq. 9 .

$$
t_{r d j}=t_{T H H}+r s t+\sum_{i=n_{H}}^{N} \frac{1}{N-n_{H}+1} t i j(x(i), y(i), x(1), y(1))
$$


After the average $R T T$ of each of the $k$ stops without repetition, $k=1,2, \ldots, P$, has been computed, the final $R T T$ can simply be computed by using Eq. 4 .

The next section is a demonstration on two cases, namely multiple floor application and nonuniform population. The vertical travel time between the ground floor and the first destination floor or between the last destination floor and the ground floor is neglected because time for both vertical travels, up and down, is constant for all scenarios. The elevator car is always assumed to start at the riser on the first destination floor and end at the riser of the last destination floor, i.e. the departing floor.

\section{CASE DEMONSTRATION}

Two cases are demonstrated, one on multi-floor round trips and one on non-uniform population distribution for easy understanding, to illustrate how the "calculation" method can be applied to the analytical traffic analysis of a general 3-D elevator system. For both cases, the time for the elevator car to travel from the ground floor to the first destination floor along the vertical riser and that from the final destination floor, i.e. the departing floor, back to the ground floor along the same or a second riser are neglected because both of them are constant under all scenarios. Also, since passenger transfer time is also constant once the rated car capacity has been fixed, it is also neglected. However, with the existence of the second group of vertical hoistways, time to travel on the ground floor from the second riser back to the first riser must be considered because the main terminal is always assumed to be close to the first group of vertical hoistways as shown in Fig. 1 and 2. Table 3 shows parameters used in the calculation.

Table 3 Values of parameters for simulation and calculation

\begin{tabular}{|l|l|l||l|l|l|}
\hline Description & Symbol & Value & Description & Symbol & Value \\
\hline \hline horizontal $90^{\circ}$ transition time & $t_{T H H}$ & $6 \mathrm{~s}$ & door opening time & $t_{o}$ & $2 \mathrm{~s}$ \\
\hline vertical $90^{\circ}$ transition time & $t_{T H V}$ & $10 \mathrm{~s}$ & $\begin{array}{l}\text { door closing time including } \\
\text { dwell time }\end{array}$ & $t_{c}$ & $6 \mathrm{~s}$ \\
\hline rated vertical speed & $v_{v}$ & $1 \mathrm{~m} / \mathrm{s}$ & $\begin{array}{l}\text { travel time between riser and } \\
\text { stop } R(i, 1,1)\end{array}$ & $r s t$ & $10 \mathrm{~s}$ \\
\hline rated horizontal speed & $v_{h}$ & $2 \mathrm{~m} / \mathrm{s}$ & $\begin{array}{l}\text { vertical acceleration and } \\
\text { deceleration time }\end{array}$ & $t_{v}(1)$ & $9 \mathrm{~s}$ \\
\hline $\begin{array}{l}\text { horizontal acceleration and } \\
\text { deceleration time }\end{array}$ & $t_{h}(1)$ & $8 \mathrm{~s}$ & $\begin{array}{l}\text { distance traveled during } \\
\text { acceleration and deceleration }\end{array}$ & $d_{h}(1)$ & $8 \mathrm{~m}$ \\
\hline $\begin{array}{l}\text { distance between stops along a } \\
\text { column }\end{array}$ & $d_{f c}=d_{h}$ & $10 \mathrm{~m}$ & $\begin{array}{l}\text { distance between stops along a } \\
\text { row }\end{array}$ & $d_{f r}=d_{h}$ & $10 \mathrm{~m}$ \\
\hline floor height & $d_{v}$ & $5 \mathrm{~m}$ & & & \\
\hline
\end{tabular}




\subsection{Multiple Floor Application}

Three floors of a building, namely $5 / \mathrm{F}, 6 / \mathrm{F}$ and $7 / \mathrm{F}$ respectively, grouped as one zone is served by one elevator car with a rated capacity of 5 passengers. Overall, there are $(M=3$ along each row) $\mathrm{x}(Q=2$ along each column $)=6$ stops on each floor. The physical addresses of the six stops are $(i, 1,1),(i, 1,2),(i, 2,1),(i, 2,2),(i, 3,1)$ and $(i, 3,2)$ respectively, with $i=5$, 6 or 7 . The possible number of passengers at each stop is 5 , i.e. every stop uniformly populated while the total population of the three floors is 6 (stops per floor) $\mathrm{x} 5$ (passengers per stop) $\mathrm{x} 3$ (floors) $=90$. In other words, there is a possibility that all five passengers in one round trip exit at the same stop, though the chance being very remote.

The scanned sequence number is from " 1 " to " 3 × $6=18$ ". There are two different designs with the vertical riser, single and dual respectively. For single design, one group of vertical hoistways close to stop $(1,1)$ of every floor is installed. For dual design, besides the first group, another group is installed close to stop $(3,2)$. Hence, for single design, the scanned route at every floor is always from $(1,1)$ to $(1,2)$ to $(2,1), \ldots$. , until $(3,2)$. That means, after the last passenger of that particular floor has alighted from the elevator, the elevator has to travel back to $(1,1)$ and then enter the single vertical riser to get to a higher floor. At the highest floor, after all passengers have alighted from the elevator, it has to travel back to $(1,1)$ and take the same vertical riser back to the ground floor.

For dual design, after the last passenger of the 5/F has alighted from the elevator, it will travel to $(3,2)$ and take the second riser to a higher floor, either to the $6 / \mathrm{F}$ or to the $7 / \mathrm{F}$ depending on the destinations of the remaining passengers. At $6 / \mathrm{F}$, the scanned sequence is reversed, i.e. from $(3,2)$ to $(3,1)$ to $(2,1)$ to $(2,2)$ to $(1,2)$ and then to $(1,1)$. At $7 / F$, the scanned sequence is restored back to normal, same as that at $6 / \mathrm{F}$. Table 4 shows the physical address of each stop and its corresponding scanned sequence number.

Table 4 Physical addresses vs scanned sequence numbers for both vertical riser design

\begin{tabular}{|l|c|c||l|c|c|}
\hline $\begin{array}{l}\text { Physical } \\
\text { address }\end{array}$ & $\begin{array}{l}\text { Sequence } \\
\text { number of } \\
\text { single riser }\end{array}$ & $\begin{array}{l}\text { Sequence } \\
\text { number of } \\
\text { dual risers }\end{array}$ & $\begin{array}{l}\text { Physical } \\
\text { address }\end{array}$ & $\begin{array}{l}\text { Sequence } \\
\text { number of } \\
\text { single riser }\end{array}$ & $\begin{array}{l}\text { Sequence } \\
\text { number of } \\
\text { dual risers }\end{array}$ \\
\hline \hline$(5,1,1)$ & 1 & 1 & $(6,2,1)$ & 10 & 9 \\
\hline$(5,1,2)$ & 2 & 2 & $(6,3,1)$ & 11 & 8 \\
\hline$(5,2,2)$ & 3 & 3 & $(6,3,2)$ & 12 & 7 \\
\hline$(5,2,1)$ & 4 & 4 & $(7,1,1)$ & 13 & 13 \\
\hline$(5,3,1)$ & 5 & 5 & $(7,1,2)$ & 14 & 14 \\
\hline$(5,3,2)$ & 6 & 6 & $(7,2,2)$ & 16 & 15 \\
\hline$(6,1,1)$ & 7 & 12 & $(7,2,1)$ & 16 & 16 \\
\hline$(6,1,2)$ & 8 & 11 & $(7,3,1)$ & 17 & 17 \\
\hline$(6,2,2)$ & 9 & 10 & $(7,3,2)$ & 18 & 18 \\
\hline
\end{tabular}


The rated capacity of the elevator car is varied between 4, 5 and 6 . By calculation, the results are tabulated in Table 5.

Table 5 Results of RTT calculation of the multi-floor 3-D elevator system for demonstration

\begin{tabular}{|l|l|l|l|l|}
\hline $\begin{array}{l}\text { Rated car } \\
\text { capacity }\end{array}$ & $\begin{array}{l}\text { Average } \\
\text { number } \\
\text { of stops }\end{array}$ & $\begin{array}{l}\text { Stopping positions of the 5 } \\
\text { passengers on average, in scanned } \\
\text { sequence numbers }\end{array}$ & $\begin{array}{l}\text { RTT (single } \\
\text { riser) }\end{array}$ & $\begin{array}{l}\text { RTT (dual } \\
\text { risers) }\end{array}$ \\
\hline 4 & 3.68 & $4.12,7.69,11.29,14.87$ & $330.33 \mathrm{~s}$ & $331.21 \mathrm{~s}$ \\
\hline 5 & 4.47 & $3.52,6.50,9.50,12.50,15.48$ & $360.32 \mathrm{~s}$ & $355.05 \mathrm{~s}$ \\
\hline 6 & 5.23 & $3.11,5.64,8.21,10.77,13.34,15.90$ & $384.79 \mathrm{~s}$ & $373.85 \mathrm{~s}$ \\
\hline
\end{tabular}

From Table 5, it can be seen that the up-peak RTTs of both designs do not differ by much from each other. Which one is slighter larger very much depends on the overall geometry of the building, car capacity and stop distribution. With the consideration of construction complexity and cost, one group of vertical hoistways close to the main terminal would be good enough for such a 3-D elevator system.

\subsection{Non-uniform stop population}

Here, it means that the population of every potential stop varies, like the population of every floor varies in a 1-D traffic analysis problem. Readers are reminded that all equations derived in section 3 of this article are based on an assumption that every stop is uniformly populated. With non-uniform stop population, some trick is needed to manipulate the equations in section 3. To illustrate the method, we go back to the consideration of a single floor round trip.

Suppose there are altogether $M \times Q=N$ stops on a particular destination floor and each stop is associated with $u a(i)$ number of potential passengers, $i=1, \ldots, N$. A new list of stops, amount to the sum of all $u a$ 's has to be prepared so that every stop is associated with a single potential passenger only. However, one or more stops may share the same physical co-ordinates, $(x, y)$.

An example is shown below. Suppose $M=2$ and $Q=2$, i.e. 4 physical stops altogether. Stop $(1,1)$ has 2 potential passengers; stop $(1,2)$ has 3 potential passengers; stop $(2,2)$ has 2 potential passengers; stop $(2,1)$ has 1 potential passenger. The new list is generated and shown in Table 6.

Table 6 New list of stops under non-uniform population

\begin{tabular}{|l|l|l|l|}
\hline $\begin{array}{l}\text { New scanned sequence } \\
\text { number }\end{array}$ & Physical address & $\begin{array}{l}\text { New scanned sequence } \\
\text { number }\end{array}$ & Physical address \\
\hline \hline 1 & $(1,1)$ & 5 & $(1,2)$ \\
\hline 2 & $(1,1)$ & 6 & $(2,2)$ \\
\hline 3 & $(1,2)$ & 7 & $(2,2)$ \\
\hline 4 & $(1,2)$ & 8 & $(2,1)$ \\
\hline
\end{tabular}


Each scanned number is now associated with one potential passenger and all equations in section 3 are applicable. The only difference is that when travel time between two stops is considered, the exact physical addresses have to be used. If the car needs to travel from scanned number " 3 " to scanned number " 4 ", travel time has to be zero because they both belong to the same physical address.

The merit of this method is that if a uniform population distribution among stops is considered, it is simple to adjust all $u a$ 's = constant, say 1. Fig. 4(a) and 4(b) show two sets of results by Monte Carlo simulation and by calculation using equations in section 3 of this article.

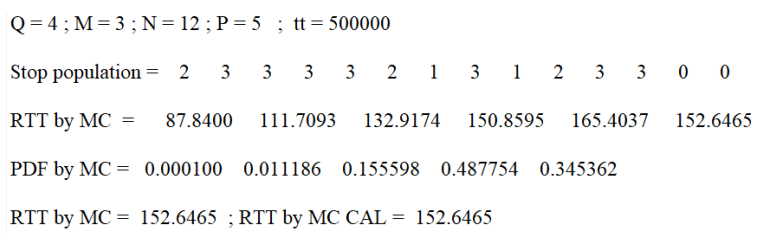

Figure 4(a) Scenario 1, $N=12$

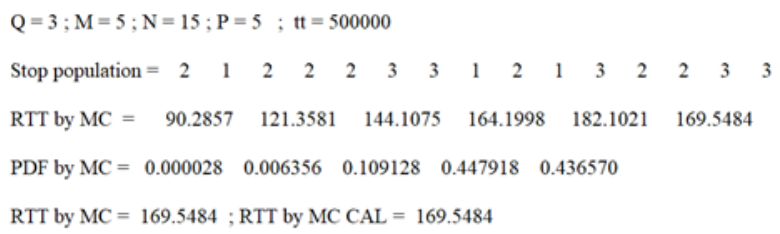

Figure 4(b) Scenario 2, $N=15$

In scenario 1 (Fig. 4(a)), there are altogether $(3 \times 4=12)$ stops and the contract capacity of the elevator is equal to 5 while the population distribution along the original scanned sequence number from 1 to 12 is listed; the first stop has 2 potential passengers; the second stop has 3 potential passengers etc. 500,000 Monte Carlo simulations were carried out and the average $R T T=152.65 \mathrm{~s}$. Statistically, the average $R T T$ by MC for one-stop journeys out of the 500,000 journeys is $87.84 \mathrm{~s}$ with a probability distribution (PDF) of 0.0001 , others being similarly. The last value of the row "RTT by MC" shows the overall average, i.e. equal to that of "RTT by MC". "RTT by MC CAL" is evaluated by the sum of products between corresponding values under "RTT by MC" and "PDF by MC". Both values agree with each other. The bottom bundle of parameters is obtained by calculation using equations in section 3 .

In scenario 2 (Fig. 4(b)), there are altogether $(5 \times 3=15)$ stops and the contract capacity of the elevator is still equal to 5 while the non-uniform population distribution along the original scanned sequence number from 1 to 15 is listed. It can be seen from both figures that the

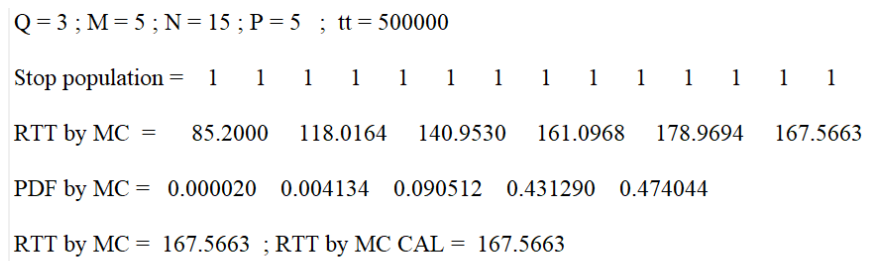

Figure 4(c) Scenario, uniform stop population 
calculated average $R T T$ 's by Monte Carlo simulation and by calculation agree well with each other. It is interesting to note that the RTT per type of stop and its associated probability distribution is very much different between the two approaches. That is because under Monte Carlo simulation, stops are on a physical address basis while under calculation, stops are on the new list of scanned sequence number basis. Their final agreement between each other strongly verifies that the calculation method is correct.

To demonstrate that this method can be applicable to a situation of uniform population. One more scenario by putting every stop associated with one potential passenger is considered, the results being shown in Fig. 4(c). Again, the two RTT's by simulation and by calculation agree well with each other. It is interesting to note that the individual RTT component and its associated probability distribution are also close to each other under two approaches. This is because under uniform population distribution, the new list of scanned sequence number is identical to the original.

\section{CONCLUSIONS}

Several approaches to the traffic analysis of a 2-D or 3-D elevator system were developed by the author of this article, jointly with others, method of which was verified by using Monte Carlo simulation. For a true 3-D elevator system, in a previous article [6], a way to estimate the up-peak RTT was achieved by linearizing a series of 2-D journeys on the destination floor by the "Scanner Method" and forming a very complicated matrix involving the origin and destination stops and this method is confined to a single floor application. At the same time, such a matrix can become very complicated as the number of stops on a floor gets larger, as well as the rated capacity of the car. Also, the designer cannot easily figure out what really happens in the complicated matrix. In a more recent article [9], a way to break down the consideration of all combinations of repeatable stops into the consideration of 1-stop, 2-stops without repetition, ..., up to $P$ separate stops ( $P$ is the number of passengers inside the car) was developed, with the help of Order Statistics. By such a process, designers can more easily understand the whole process of RTT estimation and suitably adjust the design by pure calculation. Two assumptions were made in that article, namely a single floor round trip and uniform population of every potential stop on the floor.

In this article, such two assumptions have been removed so that the up-peak $R T T$ estimation by calculation can be readily extended to cover a multiple floor 3-D trip and deal with non-uniform population of every potential stop. Of course, the original idea of linearizing a 3-D stop distribution into a 1-D line by using the "Scanner Method" is still adopted, so that stops follow a properly consecutive scanned sequence numbering policy.

When a round trip to multiple floors is considered, all stops are placed on a 1-D line with proper scanned sequence numbers. Such a sequence can also take care of multiple vertical risers as demonstrated in section 4.1. The multiple floors may not be necessarily contiguous but grouping contiguous floors into one zone seems to be one common industrial approach in destination group control systems [10]. Then, the Order Statistics based up-peak RTT can be calculated. When a non-uniform population distribution of stops is considered, each passenger of a particular stop is given a unique scanned sequence number but all passengers belonging to the same stop share the same physical address. Then, the Order Statistics based up-peak RTT 
can also be estimated. These two new approaches have been validated by using Monte Carlo simulation with great accuracy and demonstrated separately in section 4 of this article for easy understanding. Practically, the two approaches must be mixed together to arrive at the result.

It is hoped that this approach of Order Statistics based calculation method is generic enough to deal with a 3-D elevator system involving non-uniform population distribution and multi-floor application. Monte Carlo simulation, as its name tells, is still a simulation though its results' fluctuation is often much less than that of a real-time computer simulation. After all, a simulation is based on random sampling. But the beauty of calculation is that it can always return a unique result. By using the methods presented in this article, the conventional practice of doing traffic analysis by an initial calculation followed by computer simulation, also recommended by the new ISO 8100-32:2020, can still be maintained even for a 3-D elevator system. This approach could allow designers adjust their design to fit the practical environment in a much easier way.

\section{REFERENCES}

[1] Thyssenkrupp (2014), "MULTI achieves the elevator industry's holy grail”, Elevation, Issue 81, April, pp 50-53.

[2] So A and Chan W L. (2009), Intelligent Building Systems Enhanced Edition. Johnson Controls Asia, 2009, Hong Kong.

[3] So A., Al-Sharif L. and Hammoudeh A. (2015), "Traffic analysis of a simplified twodimensional elevator system", Building Services Engineering Research \& Technology, CIBSE, Vol. 36, No. 5, September, pp. 567-579. https://doi.org/10.1177/0143624414568728

[4] So A., Al-Sharif L. and Hammoudeh A. (2016), "Concept design and derivation of the round-trip time for a general two-dimensional elevator traffic system", Journal of Building Engineering, Vol. 5, March, pp. 165-177. https://doi.org/10.1016/j.jobe.2015.12.006

[5] So A, Al-Sharif L and Hammoudeh A T. (2014), "Analysis of possible twodimensional elevator traffic systems in large buildings", Elevator Technology 20, Proceedings of Elevcon 2014, Paris, France, The International Association of Elevator Engineers, July, pp. 51-61.

[6] So A., Al-Sharif L. and Hammoudeh A. (2018), "Traffic analysis of a three-dimensional elevator system", Building Services Engineering Research and Technology (first published online in May, 2017), Vol. 39, No. 1, pp. 5-20.

https://doi.org/10.1177/0143624417710106

[7] Al-Sharif L., So A., AlZoubi Q., Atallah T., Hijazi A. A. G. and Hammoudeh, A. T. (2016), "Collision prevention procedure for a rectangular two-dimensional elevator traffic system using graphical methods", Elevator Technology 21, Proceedings of Elevcon 2016, Madrid, Spain, The International Association of Elevator Engineers, pp. 14-25.

[8] Hammoudeh A. (2019), "Route selection for a three-dimensional elevator using deep reinforcement learning", Building Services Engineering, Research and Technology (first published online in September, 2019). https://doi.org/10.1177/0143624419876079

[9] So A., Al-Sharif L. and Chan W.L. (2020), "Analytical round-trip time estimation of a three-dimensional elevator system based on exact stop position identification", Journal of Building Engineering (first published online in April, 2020), Vol. 31, September. https://doi.org/10.1016/j.jobe.2020.101390 
[10] Christy T.M. (2013), "The value of contiguous grouping within destination dispatching", Elevator World, March, pp. 38-44.

\section{BIOGRAPHICAL DETAILS}

Ir Dr. Albert So is an executive board member and scientific advisor of the International Association of Elevator Engineers (IAEE). He is an honorary Visiting Professor at the Faculty of Arts, Science and Technology of University of Northampton in the U.K. He serves on the Advisory Group of Elevator World, Inc., and is based in Seattle. 